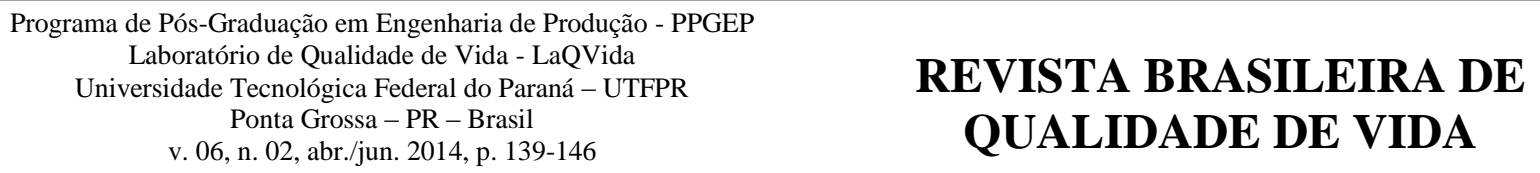

\section{REVISTA BRASILEIRA DE QUALIDADE DE VIDA}

\title{
Avaliação do controle glicêmico de pacientes diabéticos participantes de Associação de Apoio à Doença em São Lourenço do Oeste - SC
}

\section{Evaluation of glycemic control in diabetic patients participating in a Disease Support Association in São Lourenço do Oeste - SC}

\author{
Kelly Cristina Maria \\ Universidade Comunitária da Região de Chapecó - UNOCHAPECÓ - Chapecó - Santa Catarina - Brasil \\ keydrylly@ hotmail.com \\ Juliana Cristina Schmidt \\ Universidade Comunitária da Região de Chapecó - UNOCHAPECÓ - Chapecó - Santa Catarina - Brasil \\ jcs@unochapeco.edu.br
}

\section{RESUMO}

OBJETIVO: Avaliar o controle glicêmico realizado por pacientes com diabetes mellitus atendidos na Associação de Diabéticos de São Lourenço do Oeste - SC.

METODOLOGIA: Vinte e sete pacientes diabéticos de ambos os sexos foram avaliados em um estudo transversal. Os dados socioeconômicos, de estilo de vida, tratamento e características da doença foram obtidos por meio de questionário. A partir de pesquisa no banco de dados da Associação de Diabéticos foram levantados os valores de glicemia em jejum, pressão arterial, hemoglobina glicada e índice de massa corporal (IMC).

RESULTADOS: Os participantes apresentaram média de idade de 70 anos, sendo a maioria casada, com ensino fundamental (completo ou incompleto) e renda de 1 a 2 salários mínimos ao mês. Embora todos tenham relatado fazer algum tipo de dieta alimentar e uso de medicamentos, e a maioria tenha declarado realizar atividade física uma a duas vezes na semana, o controle glicêmico não se apresentou adequado. Tanto entre homens quanto mulheres, observaram-se valores médios de glicemia, hemoglobina glicada, pressão arterial sistólica e IMC acima dos valores recomendados. Somente cerca de 20,0\% dos participantes apresentou parâmetros antropométricos e de controle glicêmico adequados.

CONCLUSÃO: Embora o estado de saúde autorreferido depois da participação no grupo de apoio seja considerado bom ou muito bom, fatores relacionados à idade elevada, assim como a baixa renda e escolaridade observada nos indivíduos avaliados, podem estar dificultando o controle glicêmico adequado. Isto provavelmente prejudica a prática de atividade física e o entendimento da terapêutica, apontando para a necessidade de intensificar o aconselhamento e monitoramento destes pacientes.

PALAVRAS-CHAVE: Diabetes tipo 2. Controle Glicêmico. Grupo de apoio. 


\section{ABSTRACT}

OBJECTIVE: To evaluate glycemic control achieved by patients with diabetes mellitus attended in the Diabetics Association of Sao Lourenco do Oeste - SC.

METHODS: Twenty-seven diabetic patients from both sexes were evaluated in a cross-sectional study. The socioeconomic data, lifestyle, treatment and disease characteristics were obtained by questionnaire. From Diabetic Association database were collected fasting glucose, blood pressure, glycated hemoglobin and body mass index (BMI) values.

RESULTS: Participants mean age was 70 years, and most of them were married, with elementary school (complete or incomplete) and income 1-2 minimum wages per month. Although all patients reported to do some diet and use of medications, and the most reported to do physical activity one or two times a week, their glycemic control was not appropriated. Among both men and women was observed mean values of blood glucose, glycated hemoglobin, systolic blood pressure and BMI above recommended values. Only about $20,0 \%$ of total participants had suitable anthropometric and glycemic control parameters.

CONCLUSIONS: Although the self-reported health state after participating in the support group is considered good or very good, factors associated with older age, as well as low socio-economic and educational status observed in this population may be hindering adequate glycemic control. This probably hinders physical activity and understanding of therapy, pointing to the need for increased counseling and monitoring of these patients.

KEYWORDS: Type 2 Diabetes. Glycemic Control. Support group.

\section{Introdução}

O diabetes mellitus (DM) é definido pela Organização Mundial de Saúde (OMS) como uma síndrome de etiologia múltipla, decorrente da falta de insulina e/ou da incapacidade da mesma exercer adequadamente seus efeitos. Caracteriza-se por hiperglicemia crônica com distúrbios do metabolismo dos carboidratos, lipídios e proteínas (McLELLAN et al., 2007).

A prevalência de DM vem aumentando mundialmente. No Brasil a estimativa é de que até 2030 o número de pessoas com diabetes chegue a 439 milhões e cerca de 90,0\% sejam portadores de diabetes tipo 2, um tipo de diabetes cujo aumento vem sendo notado desde a última década (VIEGAS-PEREIRA; RODRIGUES; MACHADO, 2008; SUSO et al., 2011).

A classificação recomendada pela Sociedade Brasileira de Diabetes (SBD) inclui quatro classes clinicas do DM: DM tipo 1, DM tipo 2, outros tipos específicos de DM e DM gestacional. O DM tipo 2 (DM2) é um distúrbio multifatorial, complexo e poligênico, provocado pela combinação de vários fatores e caracterizado pela resistência periférica a insulina. O DM2 está relacionado à obesidade e ao sedentarismo, apresentando incidência elevada após os 40 anos de idade. Além disso, estima-se que 60,0 a 90,0\% dos portadores sejam obesos (RODRIGUES et al., 2010). A evolução do DM2 é a causa mais comum de cegueira, amputações e insuficiência renal em adultos no ocidente, além de aumentar a incidência de infarto agudo do miocárdio e acidente vascular cerebral (ZEVE; TOMAZ, 2011).

O tratamento inicial do DM2 compreende dieta e exercício físico, mas quando os níveis elevados de glicose persistem, o tratamento é feito com hipoglicemiantes orais e, quando em alguns casos os hipoglicemiantes orais não controlam a hiperglicemia, é necessário o uso de injeções de insulina (SMELTZER; BARE, 2002). O tratamento do DM é muito complexo e envolve mudanças no estilo de vida dos pacientes. Cuidados como a auto-monitorização da glicemia, a prática regular de atividade física, a administração de medicamentos (antidiabéticos orais e/ou insulina) e adoção de uma alimentação saudável são importantes para manter os níveis glicêmicos estabilizados e, consequentemente, prevenir as complicações crônicas que são causadas pelo aumento da glicemia (RAMOS; FERREIRA, 2011). Dessa maneira, o principal objetivo do tratamento do paciente 
diabético é a prevenção das complicações crônicas, pois a doença não é curável e sim controlável. No Brasil é bastante elevada a incidência de complicações crônicas do diabetes. Estima-se que existam cinco milhões de indivíduos portadores de diabetes, sendo que metade desconhece o diagnóstico (MAIA; CAMPOS, 2005).

Considerando-se a importância do tratamento e acompanhamento especializado dos diabéticos para o adequado controle da doença e a melhora da qualidade de vida, este estudo teve por objetivo avaliar o controle glicêmico realizado por diabéticos participantes da Associação de Diabéticos de São Lourenço do Oeste - SC.

\section{Metodologia}

O presente trabalho trata-se de um estudo transversal, descritivo e observacional para a avaliação do controle glicêmico realizado por portadores de DM, assim como para levantamento de características sócio-demográficas e relacionadas ao diabetes, de participantes de um grupo de apoio à doença em um município de pequeno porte de Santa Catarina. O projeto foi aprovado pelo Comitê de Ética em Pesquisa em Seres Humanos da Universidade Comunitária da Região de Chapecó sob nº 002/12.

\subsection{População e amostra}

A Associação de Diabéticos de São Lourenço do Oeste conta com 45 pacientes frequentadores de ambos os gêneros. Neste estudo foram avaliados 27 diabéticos, maiores de 18 anos e que realizaram exames laboratoriais periódicos para monitorização do controle glicêmico, no período entre março de 2012 a maio de 2013. Todos os pacientes foram informados previamente sobre a pesquisa e concordaram em participar da mesma por meio da assinatura do Termo de Consentimento Livre e Esclarecido (TCLE). Foram excluídos deste estudo os pacientes que não se enquadraram nos requisitos citados anteriormente.

\subsection{Coletas dos dados}

Os dados de controle glicêmico, ou seja, os valores de glicemia em jejum e de hemoglobina glicada (HbA1C), assim como de índice de massa corporal (IMC) e pressão arterial foram obtidos a partir do banco de dados da Associação de Diabéticos. Foram realizadas quatro avaliações no período da pesquisa, com exceção da HbA1C que foi verificada uma única vez.

Também foi aplicado um questionário para a coleta de informações sócio-demográficas, histórico de doenças, histórico ocupacional (estilo de vida), terapia utilizada, doenças cardiovasculares, complicações do diabetes e percepção da condição de saúde.

\subsection{Análise e interpretação dos resultados}

Os dados foram analisados no programa Epi-info 3.5.2, a fim de verificar o perfil da amostra quanto às variáveis avaliadas neste estudo. Os gráficos e tabelas foram construídos por meio do programa Microsoft Excel. Para a análise dos dados do controle glicêmico e de pressão arterial foram utilizados os valores propostos pelas Diretrizes da Sociedade Brasileira de Diabetes (SOCIEDADE BRASILEIRA DE DIABETES, 2014), enquanto que para análise do IMC foi utilizada a classificação proposta pela OMS (WORLD HEALTH ORGANIZATION, 2000).

\section{Resultados}

Participaram da pesquisa, 27 pacientes diabéticos da Associação de Diabéticos de São Lourenço do Oeste - SC, com idade variando de 56 a 87 anos e média de 70,59 anos, sendo que mais de 50,0\% apresentava idade entre 70 e 87 anos. Em relação ao gênero, $20(74,1 \%)$ pacientes 
eram do sexo feminino e 7 (25,9\%) do sexo masculino. Entre os pacientes pesquisados, observou-se que todos apresentavam renda mensal baixa, divididos entre $24(88,9 \%)$ que apresentam renda entre 1 a 2 salários mínimos e $3(11,1 \%)$ com renda mensal de 3 salários mínimos. Também se verificou um baixo nível de escolaridade entre os participantes, com $12(44,4 \%)$ apresentando o ensino fundamental incompleto e a mesma proporção tendo completado o ensino fundamental, 1 paciente $(3,7 \%)$ com o ensino médio incompleto e a mesma proporção tendo completado este nível de escolaridade, e 1 paciente (3,7\%) tendo completado o ensino superior. Quanto ao estado civil, 21 $(77,8 \%)$ dos pacientes avaliados estavam casados e $6(22,2 \%)$ viúvos.

$\mathrm{Na}$ avaliação de variáveis associadas ao estilo de vida, observou-se que $4(14,8 \%)$ dos participantes relataram não praticar nenhum tipo de atividade física, enquanto $22(81,4 \%)$ declararam praticar atividade física entre 1 e 2 vezes por semana e somente 1 paciente $(3,7 \%)$ relatou praticar atividade física 3 ou mais vezes por semana. Em relação ao consumo de bebida alcoólica, somente $6(22,2 \%)$ participantes declararam fazer uso de algum tipo de bebida alcoólica e quanto ao tabagismo pôde-se observar que 19 (70,4\%) declaram não fazer uso de tabaco, 7 (25,9\%) eram ex-fumantes e somente um $(3,7 \%)$ relatou ainda ser fumante.

A análise do perfil da doença (Tabela 1) mostrou que mais de 50,0\% dos pacientes diagnosticou a doença entre 50 e 69 anos de idade, tendo sido realizado o diagnóstico há mais de 10 anos por $25(92,6 \%)$ dos participantes. Vale ressaltar que entre as comorbidades e as complicações devido ao diabetes, a hipertensão e as doenças de retina foram as mais frequentemente autorreferidas, respectivamente. Contudo, a busca por atendimento hospitalar devido à doença foi pouco frequente na amostra avaliada.

Tabela 1 - Perfil da doença nos pacientes avaliados

\begin{tabular}{|c|c|c|}
\hline \multirow[t]{2}{*}{ Variáveis/Categorias } & Pacientes & \\
\hline & $\mathrm{N}$ & $\%$ \\
\hline \multicolumn{3}{|l|}{ Idade do diagnostico (anos) } \\
\hline Antes dos 30 anos & 1 & 3,7 \\
\hline $30-49$ & 11 & 40,7 \\
\hline $50-69$ & 15 & 55,6 \\
\hline \multicolumn{3}{|l|}{ Tempo de diagnóstico (anos) } \\
\hline Menos de 5 & 0 & 0,0 \\
\hline 5 a 10 & 2 & 7,4 \\
\hline Mais de 10 & 25 & 92,6 \\
\hline Dieta alimentar & 27 & 100,0 \\
\hline \multicolumn{3}{|l|}{ Outras doenças além do diabetes } \\
\hline Hipertensão & 21 & 77,8 \\
\hline Obesidade & 3 & 11,1 \\
\hline Doença do coração & 2 & 7,4 \\
\hline Depressão & 1 & 3,7 \\
\hline \multicolumn{3}{|l|}{ Complicações relacionadas à doença } \\
\hline Não relatado & 17 & 63,0 \\
\hline Doença renal & 1 & 3,7 \\
\hline Doença vascular & 3 & 11,1 \\
\hline Neuropatia de pés e mãos & 0 & 0,0 \\
\hline Doença da retina & 6 & 22,2 \\
\hline \multicolumn{3}{|c|}{ Internações devido à doença nos últimos 12 meses } \\
\hline Nenhuma & 26 & 96,3 \\
\hline Uma vez & 1 & 3,7 \\
\hline Mais de uma vez & 0 & 0,0 \\
\hline
\end{tabular}

Fonte: Autoria própria (2014).

$\mathrm{Na}$ avaliação do controle do diabetes realizado pelos pacientes, $23(85,2 \%)$ dos pacientes relataram controlar a doença com dieta alimentar, atividade física e medicamentos, enquanto 4 $(14,8 \%)$ controlaram o diabetes somente com medicamentos e dieta. Em particular à terapia medicamentosa, verificou-se que $100,0 \%$ dos pacientes fazem uso de algum hipoglicemiante, sendo os mais usados insulina, glibenclamida, metformina e glimepirida. Na análise dos parâmetros glicêmicos e antropométricos observou-se que $13(48,1 \%)$ apresentaram IMC maior que $30 \mathrm{~kg} / \mathrm{m}^{2} \mathrm{e}$ 
a mesma proporção apresentou sobrepeso, os valores de glicemia variaram de 66 a $351 \mathrm{mg} / \mathrm{dL}$, os valores de $\mathrm{HbA} 1 \mathrm{C}$ variaram de 5,1 a $12,9 \%$ e os valores de pressão arterial sistólica (PAS) e pressão arterial diastólica (PAD) variaram entre 90 e $200 \mathrm{mmHg}$ e 60 e $90 \mathrm{mmHg}$, respectivamente. Os mesmos parâmetros analisados separadamente nas amostras masculina e feminina (Tabela 2) revelaram que os valores médios de glicemia em jejum, hemoglobina glicada e IMC estão acima dos valores de referência para ambos os gêneros. Contudo, deve-se destacar que o IMC médio maior que $30 \mathrm{~kg} / \mathrm{m}^{2}$ indica obesidade na amostra masculina.

Tabela 2 -. Parâmetros Glicêmicos e antropométricos dos pacientes avaliados

\begin{tabular}{cccc}
\hline Variáveis & Valores recomendados & $\begin{array}{c}\text { Pacientes femininos } \\
\text { (média } \pm \text { desvio padrão) }\end{array}$ & $\begin{array}{c}\text { Pacientes masculinos } \\
\text { (média } \pm \text { desvio padrão) }\end{array}$ \\
\hline Glicemia $(\mathrm{mg} / \mathrm{dL})$ & $<110,0^{*}$ & $154,8 \pm 8,6$ & $178,1 \pm 11,6$ \\
IMC $\left(\mathrm{kg} / \mathrm{m}^{2}\right)$ & 18,5 a $24,9^{* *}$ & $29,9 \pm 3,3$ & $31,0 \pm 5,3$ \\
PAS $(\mathrm{mmHg})$ & $<130,0^{*}$ & $134,6 \pm 6,0$ & $142,1 \pm 5,5$ \\
PAD $(\mathrm{mmHg})$ & $<80,0^{*}$ & $79,2 \pm 2,5$ & $80,7 \pm 3,0$ \\
HbA1C $(\%)$ & $<7,0^{*}$ & $9,0 \pm 2,8$ & $9,9 \pm 2,8$ \\
\hline
\end{tabular}

*Valores preconizados pela Sociedade Brasileira de Diabetes (2014).
$* *$ Valores estabelecidos pela OMS (WORDL HEALTH ORGANIZATION, 2000).

Fonte: Autoria própria (2014).

Analisando-se a proporção de diabéticos com parâmetros glicêmicos e antropométricos dentro dos valores recomendados (Tabela 3), verificou-se que menos de 20,0\% e 30,0\% apresentaram glicemia e $\mathrm{HbA1C}$, respectivamente, dentro de valores que indicam um adequado controle glicêmico. Além disso, verificou-se também que menos de 30,0\% dos participantes apresentaram PAS e PAD dentro dos valores recomendados.

Tabela 3 - Proporção de diabéticos com parâmetros dentro dos valores recomendados pela SBD para adequado controle do diabetes

\begin{tabular}{ccc}
\hline Variáveis & $\mathrm{N}$ & $\%$ \\
\hline PAS (mmHg)* & $7,8 \pm 1,7$ & $28,7 \pm 6,3$ \\
PAD (mmHg)* & $6,8 \pm 4,4$ & $25,0 \pm 16,1$ \\
Glicemia (mg/dL)* & $5,0 \pm 1,6$ & $18,5 \pm 6,1$ \\
HbA1C $(\%)^{* *}$ & 5,0 & 29,4 \\
\hline
\end{tabular}

*Média e desvio-padrão obtidos a partir de avaliações em quatro momentos distintos para n=27. **Valores absolutos obtidos a partir de uma avaliação para $\mathrm{n}=17$.

Fonte: Autoria própria (2014).

Outro aspecto analisado neste estudo foi a percepção de estado de saúde relacionada à participação no grupo de apoio à doença do município. Os dados mostraram que todos os participantes relataram fazer parte da associação há mais de 5 anos, sendo que $15(55,6 \%)$ avaliaram a saúde antes de participar do grupo de apoio como boa, $11(40,7 \%)$ avaliaram como regular e $1(3,7 \%)$ como ruim. Entretanto, após o ingresso no grupo de diabéticos, 18 (66,7\%) avaliaram a saúde como muito boa e $9(33,3 \%)$ avaliaram a saúde como boa.

\section{Discussão}

Diante das características gerais do grupo avaliado, pode-se observar que a maioria dos pacientes diabéticos era do sexo feminino e uma minoria do sexo masculino. Este resultado pode ser explicado pela sobrevida do sexo feminino ser maior que a do sexo masculino, além do preconceito que os homens apresentam em procurar um atendimento médico (PINCINATO, 2003). Tavares, Drumond e Perreira (2008) descreveram um maior percentual de prevalência entre as mulheres 
(71,2\%) quando comparado aos homens $(28,8 \%)$ em um estudo de idosos com diabetes no município de Uberaba - MG.

Quando ao estado civil dos indivíduos avaliados verificou-se que a maioria estava casado (77,8\%), apresentava renda mensal entre 1 e 2 salários mínimos (88,9\%), baixo nível de escolaridade $(88,8 \%)$ e que a faixa etária predominante estava entre 70 e 86 anos de idade. Este dado foi bastante relevante, uma vez que baixa renda e escolaridade, assim como idade avançada podem dificultar o entendimento da terapêutica e dificultar a adequação a dieta e a atividade física (McLELLAN et al., 2007).

Mendes e colaboradores (2011) descrevem que o rápido envelhecimento da população, o estilo de vida, a urbanização, a dieta inadequada, o sedentarismo, o consumo de álcool e de tabaco são fatores responsáveis pelas alterações cardiovasculares que evoluem nos pacientes diabéticos. Em relação aos fatores de risco relacionados ao diabetes verificou-se a predominância de indivíduos que afirmaram seguir uma dieta alimentar, não fazer uso do tabaco e não fazer uso de bebida alcoólica. Contudo, deve-se destacar que 14,8\% dos diabéticos avaliados são sedentários e, embora $81,4 \%$ pratiquem atividade física 1 a 2 vezes na semana, a frequência está abaixo da recomendada para os benefícios no controle do diabetes que é de 3 a 5 vezes na semana em períodos de 30 a 60 minutos (SOCIEDADE BRASILEIRA DE DIABETES, 2009). A pouca prática de atividade física também pode estar contribuindo para o sobrepeso observado nesta amostra, visto que as médias dos valores de IMC, tanto para as mulheres quanto para os homens, ficou próximo ou acima de 30 $\mathrm{kg} / \mathrm{m}^{2}$ (Tabela 2). Segundo a SBD (SOCIEDADE BRASILEIRA DE DIABETES, 2009), a importância do controle de peso na redução dos riscos relacionados ao DM é de grande importância, o ganho de peso agrava a situação metabólica predispondo a outras enfermidades como a hipertensão arterial e as dislipidemias.

O perfil da doença observado no grupo de diabéticos analisado foi predominantemente de pacientes que descobriram a doença entre os 50 e 69 anos, que apresentaram como comorbidade a hipertensão e não sofreram internações nos últimos 12 meses (Tabela 1). Considera-se de suma importância o controle da pressão arterial nos pacientes diabéticos, tanto para a prevenção da doença cardiovascular quanto para minimizar a progressão da retinopatia diabética, e, segundo a SBD, sugere-se que estes indivíduos apresentem níveis de PAS abaixo de $130 \mathrm{mmHg}$ e de PAD abaixo de $80 \mathrm{mmHg}$. Neste estudo verificou-se que tanto os indivíduos do sexo masculino quanto feminino apresentaram a pressão arterial alterada (Tabela 2), o que revela maior risco para as complicações da doença nesses indivíduos. Os pacientes portadores do DM2 apresentam uma predisposição de duas a quatro vezes maior de morrer por doença cardíaca em relação a não diabéticos, e quatro vezes mais chance de ter doença vascular periférica (DVP) e acidente vascular cerebral (AVC), além de ser apontado como uma das principais causas de cegueira em adultos com idade entre 20 e 74 anos (SCHEFFEL et al., 2004).

Em relação à glicemia e aos níveis de hemoglobina glicada, pode-se observar que os dois sexos apresentaram valores acima dos recomendados segundo a SBD, indicando um inadequado manejo terapêutico na maioria dos pacientes analisados. Níveis de HbA1C acima de 7,0\% estão associados a um risco progressivamente maior de complicações crônicas. Por isso, o consenso atual de tratamento do DM define a meta de 7,0\% (ou de 6,5\%, de acordo com algumas sociedades médicas) como limite superior, acima do qual está indicada a revisão do esquema terapêutico em vigor (PIMAZONI-NETTO et al., 2009).

Além disso, é preciso destacar a baixa proporção de pacientes com parâmetros de controle do diabetes adequados (Tabela 3) observada na amostra estudada. $\mathrm{O}$ diabetes não adequadamente controlado está associado ao aumento da mortalidade e ao alto risco do desenvolvimento de complicações macrovasculares e microvasculares e neuropáticas, podendo desta forma resultar em insuficiência renal, cegueira e amputações dos membros, sendo responsável pela redução da capacidade de trabalho e da expectativa de vida (BATISTA et al., 2005). Vale ressaltar ainda que o DM traz algumas limitações e novas incumbências para as pessoas que as desenvolvem, que muitas vezes não são aceitas e superadas por falta de conhecimento de como enfrentá-las (COELHO; SILVA; PADILHA, 2009). 
A participação dos diabéticos no grupo de apoio há mais de cinco anos parece ter melhorado a percepção da própria saúde dos participantes, uma vez que todos relataram apresentar muito boa ou boa saúde, diferente da percepção regular da saúde que tinham antes de frequentar o grupo, relatada por $40,7 \%$ dos participantes. O estudo realizado em um hospital em Belo Horizonte - MG observou que pacientes que frequentam grupos educativos em diabetes melhoram seus conhecimentos e modificam atitudes sobre a doença, favorecendo a adesão à dieta e às atividades físicas, auxiliando no controle da glicemia (TORRES et al., 2009).

A idade elevada dos pacientes, assim como a baixa renda e escolaridade, podem estar afetando negativamente o controle glicêmico, devido à dificuldade que estes encontram no entendimento da terapêutica medicamentosa e, até mesmo, para a prática de atividade física com maior frequência. Vale ressaltar a importância da Associação dos Diabéticos de São Lourenço do Oeste - SC na melhoria da qualidade de vida de todos os participantes da pesquisa. Contudo, os parâmetros glicêmicos, assim como o IMC e a PAS, acima dos valores recomendados na maioria dos participantes apontam para a necessidade de intensificar $\mathrm{o}$ aconselhamento $\mathrm{e} o$ acompanhamento da atividade física, da dieta alimentar e dos hábitos de vida destes pacientes.

\section{Agradecimentos}

Agradecemos à Associação de Diabéticos de São Lourenço do Oeste - SC pela disponibilização dos dados e acesso aos pacientes participantes desta pesquisa.

\section{Referências}

BATISTA, M. C. R.; PRIORE, S. E.; ROSADO, L. E. F. P. L.; TINÔCO, A. L. A.; FRANCESCHINI, S. C. C. Avaliação dos resultados da atenção multiprofissional sobre controle glicêmico, perfil lipídico e estado nutricional de diabéticos atendidos em nível primário. Revista de Nutrição, Campinas, v.18, n.2, 2005.

COELHO, S. M.; SILVA, V.G. M. D.; PADILHA, S. I. M. Representações sociais de pé diabético para pessoas com diabetes mellitus tipo 2. Revista da Escola de Enfermagem da USP, São Paulo, v. 43, n. 1, 2009.

MAIA, S. A.C.; CAMPOS, H. A. C. Diabetes mellitus como causa de perda auditiva. Revista Brasileira de Otorrinolaringologia, São Paulo, v. 71, n. 1, 2005.

MENDES, B. A. T.; GOLDBAUM, M.; SEGRI, J. N.; BARROS, A. B. M.; CESAR, G. L. C.; CARANDINA, L.; ALVES, P. G. C. M. Diabetes mellitus: fatores associados à prevalência em idosos, medidas e praticas de controle e uso dos serviços de saúde em São Paulo, Brasil. Caderno de Saúde Pública, Rio de Janeiro, v. 27, n. 6, 2011.

McLELlAN, P. C. K.; BARBALHA, M. S.; CATTALINI, M.; LEIARIO, C. A. Diabetes mellitus tipo 2, síndrome metabólica e modificação no estilo de vida. Revista de Nutrição, Campinas, v. 20, n. 5, 2007.

PIMAZONI-NETTO, P. A.; ANDRIOLO, A.; FILHO, F. F.; TAMBASCIA, M.; GOMES, B. M.; MELO, M.; SUMITA, M. N.; LYRA, R.; CAVALCANTI, S. Atualização sobre hemoglobina glicada (HbA1c) para avaliação do controle glicêmico e para o diagnóstico do diabetes: aspectos clinicos e laboratóriais. Jornal Brasileiro de Patologia e Medicina Laboratorial, Rio de Janeiro, v. 5, n. 1, p. 31-48, fev. 2009. 
PINCINATO, E. C.; Atualização do diagnóstico, classificação para diabetes mellitus (DA). Revista Brasileira de Ciência da Saúde, São Paulo, v. 1, n. 1, p. 62-67, 2003.

RAMOS, L.; FERREIRA, P. A. E. Fatores emocionais, qualidade de vida e adesão ao tratamento em adultos com diabetes tipo 2. Revista Brasileira de Crescimento e Desenvolvimento Humano, São Paulo, v. 21, n. 3, 2011.

RODRIGUES, R; POZZONBON, A; HOERLLE, J; REMPEL, C; PÉRICO, E. Avaliação do perfil glicêmico de pacientes com diabetes mellitus tipo 2 com e sem administração de infusão de folhas de Averrhoa carambola. Revista Scientia Medica, Porto Alegre, v. 20, n. 2, p. 161-165, 2010.

SCHEFFEL, S. R.; BORTOLANZA, D.; WEBER, S. C.; COSTA, A. L.; CANANI, H. L.; SANTOS, G. K.; CRISPIM, D.; ROISENBERG, I.; LISBÔA, K. R. H.; TRES, S. G.; GROSS, L. J. Prevalência de complicações micro e macrovasculares e de seus fatores de risco em pacientes com diabetes melito do tipo 2 em atendimento ambulatorial. Revista da Associação Médica Brasileira, São Paulo, v. 50, n. 3, 2004.

SMELTZER, S. C.; BARE, B. G. Tratado de enfermagem médico-cirúrgica. Rio de Janeiro: Guanabara Koogan, 2002.

SOCIEDADE BRASILEIRA DE DIABETES. Diretrizes da Sociedade Brasileira de Diabetes 2013-2014. 4. ed. São Paulo: A. C. Farmacêutica, 2014.

SOCIEDADE BRASILEIRA DE DIABETES. Diretrizes da Sociedade Brasileira de Diabetes 2009. 3. ed. Itapevi: A. Araújo Silva Farmacêutica, 2009.

SUSO, K.; ENGROFF, P.; ELY, L.; MORIGUCHI, Y.; CARLI, G. A.; MORRONE, F. B. Prevalência de diabetes mellitus e correlação entre testes de glicemia em pacientes idosos atendidos no ambulatório do Instituto de Geriatria e Gerontologia, PUCRS. Revista Brasileira de Análises Clínicas, Rio de Janeiro, v. 43, n. 2, p. 155-159, 2011.

TAVARES, D. M. S.; DRUMOND, F. R.; PERREIRA, G. A. Condição de saúde dos idosos com diabetes no município de Uberaba, Minas Gerais. Texto \& Contexto Enfermagem, Florianópolis, v. 17, n. 2, p. 342-349, 2008.

TORRES, H. C.; FRANCO, L. J.;STRADIOTO, M.A.; HORTALE, V. A.; SCHALL, V. T. Avaliação estratégica de educação em grupo e individual no programa educativo em diabetes. Revista de Saúde Pública, Rio de Janeiro, v. 43, n. 2, p. 291-298, 2009.

VIEGAS-PEREIRA, V. F. P. A.; RODRIGUES. N. R.; MACHADO, J. C. Fatores associados à prevalência de diabetes autorreferido entre idosos de Minas Gerais. Revista Brasileira de Estudos de População, São Paulo, v. 25, n. 2, p. 365-376, 2008. rrossef

WORLD HEALTH ORGANIZATION. Obesity: Preventing and managing the global epidemic Report of a WHO consultation on obesity. WHO Technical Report Series 894. Geneva, 2000.

ZEVE, M. L. J.; TOMAZ, B. A. C. Cirurgia metabólica-cura para diabete tipo 2. Arquivos Brasileiros de Cirurgia Digestiva, São Paulo, v. 24, n. 4, 2011. 